\title{
Prevalence, Characteristics, and Clinical Significance of Concomitant Cardiomyopathies in Subjects with Bicuspid Aortic Valves
}

\author{
Hyeonju Jeong ${ }^{1}$, Chi Young Shim ${ }^{2}$, Darae Kim³ ${ }^{3}$ Jah Yeon Choi ${ }^{2}$, Kang-Un Choi ${ }^{2}$, \\ Soo Youn $\mathrm{Lee}^{4}$, Geu-Ru Hong ${ }^{2}$, and Jong-Won $\mathrm{Ha}^{2}$ \\ ${ }^{1}$ Division of Cardiology, Department of Internal Medicine, Myongji Hospital, Hanyang University College of Medicine, Goyang; \\ ${ }^{2}$ Division of Cardiology, Department of Internal Medicine, Severance Cardiovascular Hospital, Yonsei University College of Medicine, Seoul; \\ ${ }^{3}$ Department of Medicine, Heart Vascular Stroke Institute, Samsung Medical Center, Sungkyunkwan University School of Medicine, Seoul; \\ ${ }^{4}$ Division of Cardiology, Department of Internal Medicine, Sejong General Hospital, Bucheon, Korea.
}

\begin{abstract}
Purpose: The present study aimed to investigate the prevalence, characteristics, and clinical significance of concomitant specific cardiomyopathies in subjects with bicuspid aortic valves (BAVs).

Materials and Methods: A total of 1186 adults with BAV (850 males, mean age 56 \pm 14 years) at a single tertiary center were comprehensively reviewed. Left ventricular non-compaction, hypertrophic cardiomyopathy, and idiopathic dilated cardiomyopathy were confirmed when patients fulfilled current clinical and echocardiographic criteria. Clinical and echocardiographic characteristics, including comorbidities, heart failure presentation, BAV morphology, function, and aorta phenotypes, in BAV subjects with or without specific cardiomyopathies were compared.

Results: Overall, 67 subjects (5.6\%) had concomitant cardiomyopathies: 40 (3.4\%) patients with left ventricular non-compaction, 17 (1.4\%) with hypertrophic cardiomyopathy, and $10(0.8 \%)$ with dilated cardiomyopathy. BAV subjects with hypertrophic cardiomyopathy had higher prevalences of diabetes mellitus and heart failure with preserved ejection fraction, and tended to have type 0 phenotype, while BAV subjects with dilated cardiomyopathy showed higher prevalences of chronic kidney disease and heart failure with reduced ejection fraction. BAV subjects with left ventricular non-compaction were significantly younger and predominantly male, and had greater BAV dysfunction and a higher prevalence of normal aorta shape. In multiple regression analysis, cardiomyopathy was independently associated with heart failure (odds ratio $2.795,95 \%$ confidential interval $1.603^{-}$ 4.873, $p<0.001$ ) after controlling for confounding factors.

Conclusion: Concomitant cardiomyopathies were observed in $5.6 \%$ of subjects with BAV. A few different clinical and echocardiographic characteristics were found. The presence of cardiomyopathy was independently associated with heart failure.
\end{abstract}

Key Words: Bicuspid aortic valve, hypertrophic cardiomyopathy, non-compaction, dilated cardiomyopathy

Received: March 25, 2019 Revised: June 28, 2019

Accepted: July 15, 2019

Corresponding author: Chi Young Shim, MD, PhD, Division of Cardiology, Department of Internal Medicine, Severance Cardiovascular Hospital, Yonsei University College of Medicine, 50-1 Yonsei-ro, Seodaemun-gu, Seoul 03722, Korea.

Tel: 82-2-2228-8453, Fax: 82-2-2227-7742, E-mail: cysprs@yuhs.ac

-The authors have no potential conflicts of interest to disclose.

(C) Copyright: Yonsei University College of Medicine 2019

This is an Open Access article distributed under the terms of the Creative Commons Attribution Non-Commercial License (https://creativecommons.org/licenses/ by-nc/4.0) which permits unrestricted non-commercial use, distribution, and reproduction in any medium, provided the original work is properly cited.

\section{INTRODUCTION}

Bicuspid aortic valve (BAV) is the most common congenital heart valve disease, with an overall incidence of approximately $1 \%$ in the general population. ${ }^{1,2}$ Subjects with BAV often present with aortic dilatation and may exhibit mechanical functional alterations in vasculatures. ${ }^{3,4}$ In addition, BAV is a highly heritable trait, often associated with other congenital heart defects or genetic syndromes. ${ }^{5,6}$

Regarding myocardial characteristics in subjects with BAV, most previous studies have focused on subclinical left ven- 
tricular dysfunction associated with increased aortic stiffness..$^{7-9}$ Although a possible association between BAV and specific cardiomyopathies (CMs) based on common genetic traits has been proposed in several case reports, ${ }^{10,11}$ data on the prevalence of coexisting specific CMs in subjects with BAV are limited. In a previous study, an incidence of $11 \%$ for left ventricular non-compaction (LVNC) in 109 patients with BAV was reported. ${ }^{12}$ In a recent large population study, a prevalence of only $0.4 \%$ for hypertrophic CM (HCM) was reported in 5430 patients with BAV, similar to the general population..$^{13}$ However, the prevalence of concomitant specific CMs might be different based on ethnicity. Moreover, data are lacking regarding clinical and echocardiographic characteristics based on the type of concomitant CMs. Therefore, in the present study, the prevalence, characteristics, and clinical significance of concomitant CMs, including LVNC, HCM, and idiopathic dilated CM (DCM), were determined using a large Korean BAV registry.

\section{MATERIALS AND METHODS}

\section{Study population}

We retrospectively reviewed subjects diagnosed with BAV using transthoracic echocardiography in Severance Cardiovascular Hospital from January 2003 to December 2017. A total of 1186 subjects ( 850 males, mean age $56 \pm 14$ years) were included in this study. All echocardiographic studies in subjects with BAV were manually reviewed for confirmation. Patient medical data as recorded by the physicians were carefully reviewed by two experienced observers who were blinded to echocardiography results. Patients who presented with acute heart failure were not included in the present study. The institutional review board of Severance Hospital approved the present study (IRB No. 2015-0403-001), which was conducted in compliance with the Declaration of Helsinki. The subjects were classified into four groups based on the presence of specific CMs.

Standard two-dimensional and Doppler measurements were performed following the American Society of Echocardiography guidelines..$^{14} \mathrm{BAV}$ was diagnosed based on anatomic evaluation of the aortic valve, when only two cusps were unequivocally identified in systole and diastole in the short-axis view and with a clear "fish mouth" appearance during systole. ${ }^{15} \mathrm{BAV}$ morphology was classified into four types based on position and pattern of raphe and cusps: Type 1 exhibited fusion of the left coronary and right coronary cusps, type 2 indicated fusion of the right coronary and noncoronary cusps, and type 3 comprised fusion of the left coronary and noncoronary cusps. Type 0 was recorded when there were two developed cusps and no raphe (true type). ${ }^{15,16}$ The severity of aortic stenosis or aortic regurgitation was assessed using integrated approaches. ${ }^{17,18}$ The dimensions of the sinus of Valsalva, sinotubular junction, and ascending aorta were measured as previously described. ${ }^{7,15}$ The presence of aortopathy was defined as an ascending aorta dimension $\geq 40 \mathrm{~mm}$, as previously defined.

HCM was clinically diagnosed based on the presence of unexplained myocardial hypertrophy (wall thickness $\geq 15 \mathrm{~mm}$ ) in the absence of local or systemic etiologies capable of producing the extent of hypertrophy evident. ${ }^{13}$ Mild systemic hypertension was not an exclusion criterion in the diagnosis of HCM. Subjects with coexisting HCM were subdivided into three morphologically obstructive HCM subgroups, non-obstructive HCM, non-apical HCM, or non-obstructive apical HCM, based on either the presence of left ventricular outflow track obstruction or the predominant hypertrophy at the left ventricular apex. LVNC was diagnosed based on previously suggested echocardiographic criteria ${ }^{19-21}$ and/or ratios between noncompacted and compacted layers of the left ventricular wall on cardiac magnetic resonance imaging. ${ }^{22}$ If the diagnosis of LVNC was suspected but not confirmed using echocardiography, cardiac magnetic resonance imaging was performed. DCM was defined as an ejection fraction $<40 \%$ in the presence of increased left ventricular dimension. Subjects with ischemic heart disease, uncorrected or corrected severe aortic stenosis or aortic regurgitation, and other reversible causes were excluded from diagnosis of idiopathic DCM in this study. Echocardiographic data were gathered and analyzed by experienced sonographers blinded to each patient's clinical data. Heart failure was diagnosed using current diagnostic criteria ${ }^{23}$ and was categorized based on left ventricular ejection fraction (LVEF) as follows: heart failure with preserved ejection fraction (LVEF $\geq 50 \%$ ) and heart failure with reduced ejection fraction (LVEF $<50 \%)$.

Continuous variables are expressed as a mean \pm standard deviation. Categorical variables are expressed as a number (percentage). Comparisons between groups were performed using standard $\lambda^{2}$ tests for categorical variables and paired $t$-tests for continuous variables. Univariate and multivariate logistic regression analyses were performed. All statistical analyses were performed using SPSS Statistics, version 23.0 (IBM, Armonk, NY, USA). $p$ values $<0.05$ were considered statistically significant.

\section{RESULTS}

\section{Prevalence of coexistent CMs in BAV subjects}

Overall, 67 subjects (5.6\%) had concomitant CMs: $10(0.8 \%)$ subjects with DCM, 17 (1.4\%) with HCM, and 40 (3.4\%) with LVNC. Among the subjects with coexistent HCM, five had obstructive HCM, six presented with non-obstructive HCM, and six with apical HCM (Fig. 1).

\section{Characteristics of BAV subjects with HCM, LVNC, or DCM}

Baseline characteristics of the subjects with or without specific CMs are shown in Table 1. Subjects with DCM had a higher 


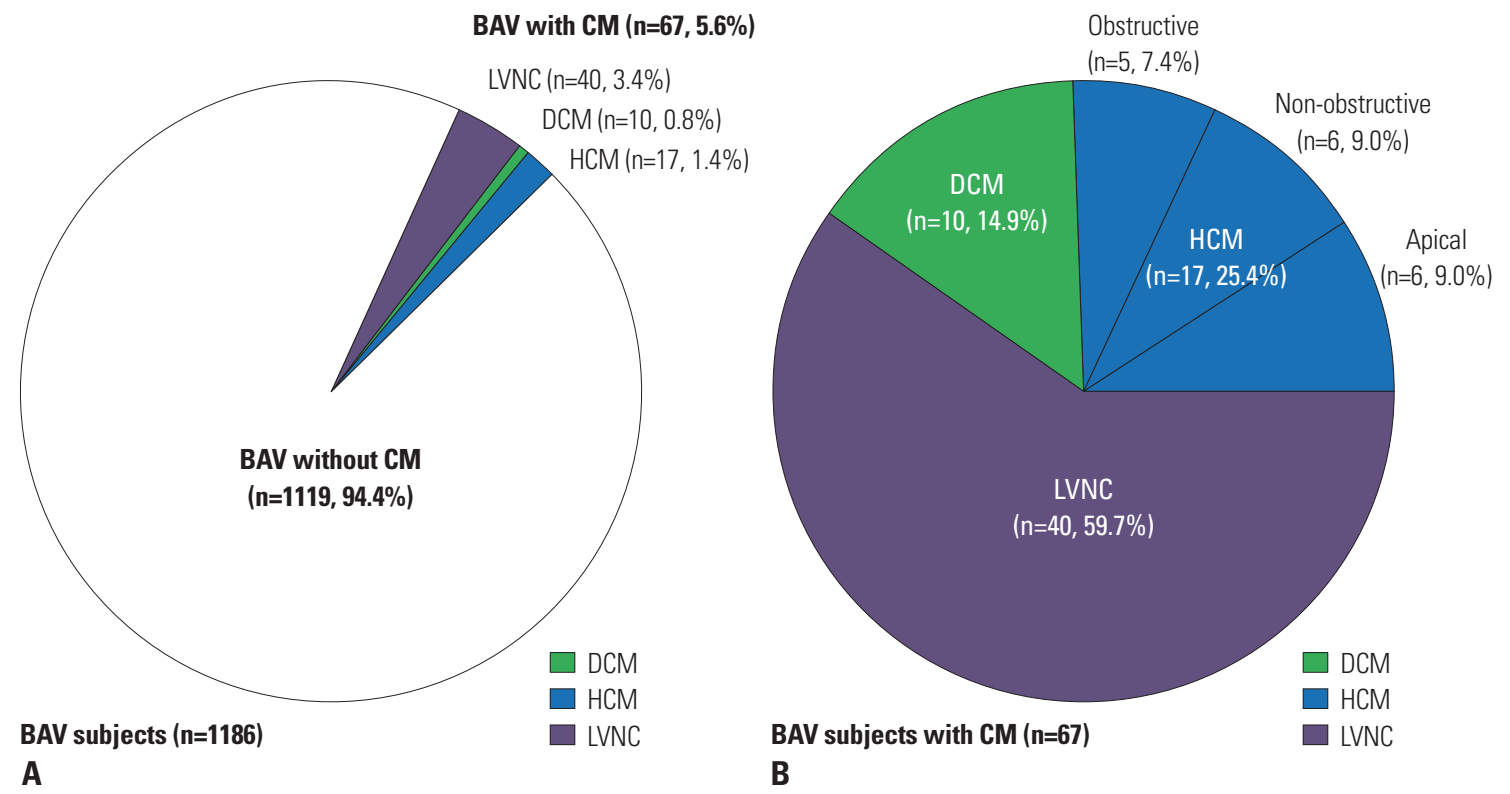

Fig. 1. Prevalence of specific cardiomyopathies in bicuspid aortic valves (BAV) subjects. (A) A total of 1186 BAV subjects. (B) Sixty-seven BAV subjects with cardiomyopathy (CM). LVNC, left ventricular non-compaction; DCM, dilated cardiomyopathy; HCM, hypertrophic cardiomyopathy.

Table 1. Baseline Characteristics according to the Presence of Specific Cardiomyopathy

\begin{tabular}{|c|c|c|c|c|c|}
\hline & No CM (n=1119) & $\operatorname{DCM}(n=10)$ & HCM $(n=17)$ & LVNC (n=40) & $p$ value \\
\hline Age (yr) & $56 \pm 15$ & $56 \pm 5$ & $60 \pm 13$ & $51 \pm 16^{* \neq}$ & 0.097 \\
\hline Male & $793(71)$ & $8(80)$ & $13(77)$ & $35(88)^{*}$ & 0.135 \\
\hline Body mass index $\left(\mathrm{kg} / \mathrm{m}^{2}\right)$ & $23.9 \pm 3.6$ & $23.7 \pm 3.5$ & $24.7 \pm 2.2$ & $23.4 \pm 3.2$ & 0.666 \\
\hline Hypertension & $462(41)$ & $3(30)$ & $10(59)$ & $17(43)$ & 0.444 \\
\hline Diabetes mellitus & $209(19)$ & $3(30)$ & $9(52)^{*}$ & $4(10)^{\ddagger}$ & 0.001 \\
\hline Dyslipidemia & $231(21)$ & $1(10)$ & $3(18)$ & $10(25)$ & 0.742 \\
\hline CAD & $182(16)$ & $0(0)$ & $2(12)$ & $5(13)$ & 0.464 \\
\hline CKD & $97(9)$ & $3(30)^{*}$ & $2(12)$ & $4(10)$ & 0.124 \\
\hline Heart failure & $178(16)$ & $6(60)^{*}$ & $8(47)$ & $8(20)^{\ddagger \ddagger}$ & $<0.001$ \\
\hline Preserved EF & $129(12)$ & $0(0)$ & $8(47)^{*+}$ & $6(15)^{\ddagger}$ & $<0.001$ \\
\hline Reduced EF & $49(4)$ & $6(60)^{*}$ & $0(0)^{\dagger}$ & $2(5)^{\dagger}$ & $<0.001$ \\
\hline
\end{tabular}

$\mathrm{CM}$, cardiomyopathy; DCM, dilated cardiomyopathy; HCM, hypertrophic cardiomyopathy; LVNC, left ventricular non-compaction; CAD, coronary artery disease; CKD, chronic kidney disease; EF, ejection fraction.

Values are presented as mean \pm standard deviation or $\mathrm{n}(\%)$ unless otherwise indicated.

${ }^{*} p<0.05$ compared to the group with no $\mathrm{CM} ;{ }^{\dagger} p<0.05$ compared to the DCM group; ${ }^{\ddagger} p<0.05$ compared to the HCM group.

prevalence of chronic kidney disease and heart failure with reduced ejection fraction, compared with those without $\mathrm{CM}$. Subjects with HCM showed higher prevalences of diabetes mellitus and heart failure with preserved ejection fraction than those without CM. Subjects with LVNC were younger and predominantly male, compared with those without CM. In addition, subjects with LVNC exhibited a lower prevalence of heart failure than patients with DCM or HCM.

Table 2 shows the structural and functional characteristics of the left ventricle in each group. The echocardiographic variables, including left ventricle dimension, wall thickness, and LVEF, were significantly different among the groups because of their own disease characteristics. LA volume index values in BAV subgroups with specific CMs were significantly greater than those in the BAV group without CMs. Early diastolic mitral annular tissue (e') velocity and ratio of early diastolic mitral inflow velocity to e' velocity (E/e') in BAV subgroups with HCM and DCM were also significantly greater than those in BAV subgroups without CMs. However, right ventricular systolic pressure was not significantly different among the groups.

The BAV characteristics in Table 3 and Fig. 2 show that the type $1 \mathrm{BAV}$ phenotype (fusion of right and left coronary cusps) was the most prevalent morphology in all groups. Although subjects with HCM tended to have type 0 phenotype, there was no statistically significant differences in BAV phenotypes among the groups. Subjects with DCM comprised a higher prevalence of no or mild dysfunction, while subjects with LVNC exhibited a lower prevalence of no or mild dysfunction. Regarding aorta 
phenotypes, subjects with LVNC revealed a higher prevalence of normal shape than those without CMs. Although statistical significance was not observed, more DCM patients had predominant AA phenotype, and none showed predominant sinus of Valsalva. Fig. 3 illustrates the representative cases of coexisting CMs in subjects with BAV.

\section{Clinical significance of concomitant CMs in BAV}

Logistic regression analysis was performed to investigate factors associated with heart failure in subjects with BAV. In univariate analysis, increased age was significantly associated with heart failure [odds ratio (OR) 1.035, $p<0.001$ ], while gender was not. Comorbidities, such as hypertension (OR 1.793, $p<0.001$ ), diabetes mellitus (OR 1.704, $p=0.003$ ), and chronic kidney disease (OR 2.226, $p<0.001$ ), were significantly associated with heart failure. Aortic valve dysfunction was not a significant associating factor for heart failure. However, an increased aorta diameter (OR 1.020, $p=0.046$ ) was significantly associated with heart failure. Moreover, presence of $\mathrm{CM}$ was the strongest factor associated with heart failure (OR 2.582, $p<0.001)$. In multivariate analysis, age, hypertension, chronic kidney disease, and presence of concomitant CM were independently associ-

Table 2. Left Ventricle Characteristics according to the Presence of Specific Cardiomyopathy

\begin{tabular}{|c|c|c|c|c|c|}
\hline & No CM (n=1119) & $\mathrm{DCM}(\mathrm{n}=10)$ & HCM (n=17) & LVNC $(n=40)$ & $p$ value \\
\hline LVEDD (mm) & $53.5 \pm 9.1$ & $68.7 \pm 10.3^{*}$ & $50.7 \pm 9.9^{\dagger}$ & $59.0 \pm 10.2^{*+\neq}$ & $<0.001$ \\
\hline LVESD (mm) & $36.3 \pm 8.7$ & $59.2 \pm 9.8^{*}$ & $31.9 \pm 8.1^{* \dagger}$ & $41.9 \pm 9.5^{* \neq \ddagger}$ & $<0.001$ \\
\hline IVS thickness (mm) & $10.7 \pm 2.2$ & $10.1 \pm 2.6$ & $14.7 \pm 4.2^{* \dagger}$ & $10.7 \pm 2.3^{\ddagger}$ & $<0.001$ \\
\hline PW thickness (mm) & $10.5 \pm 2.0$ & $10.3 \pm 2.2$ & $12.2 \pm 2.3^{*+}$ & $10.7 \pm 2.0^{\ddagger}$ & 0.005 \\
\hline LV ejection fraction (\%) & $63 \pm 12$ & $29 \pm 11^{*}$ & $70 \pm 8^{*+}$ & $58 \pm 12^{*+\neq}$ & $<0.001$ \\
\hline $\mathrm{LA}$ volume index $\left(\mathrm{mL} / \mathrm{m}^{2}\right)$ & $32.0 \pm 16.4$ & $46.9 \pm 21.4^{*}$ & $36.0 \pm 14.4$ & $37.7 \pm 16.4^{*}$ & 0.004 \\
\hline $\mathrm{e}^{\prime}$ velocity (cm/s) & $6.1 \pm 2.5$ & $3.5 \pm 1.4^{*}$ & $4.1 \pm 1.6^{*}$ & $6.7 \pm 3.0^{\dagger \neq}$ & $<0.001$ \\
\hline$E / e^{\prime}$ & $13.0 \pm 5.9$ & $20.0 \pm 6.6^{*}$ & $17.5 \pm 7.3^{*}$ & $12.8 \pm 5.4^{\dagger \ddagger}$ & $<0.001$ \\
\hline $\operatorname{RVSP}(\mathrm{mm} \mathrm{Hg})$ & $29 \pm 11$ & $33 \pm 12$ & $25 \pm 5$ & $29 \pm 10$ & 0.526 \\
\hline
\end{tabular}

CM, cardiomyopathy; DCM, dilated cardiomyopathy; HCM, hypertrophic cardiomyopathy; LVNC, left ventricular non-compaction; LVEDD, left ventricular end-diastolic dimension; LVESD, left ventricular end-systolic dimension; IVS, interventricular septum; PW, posterior wall; LV, left ventricle; LA, left atrium; e', early diastolic mitral annular tissue; E/e', ratio of early diastolic mitral inflow velocity to early diastolic mitral annular tissue velocity; RVSP, right ventricular systolic pressure.

Values are presented as mean \pm standard deviation unless otherwise indicated.

${ }^{*} p<0.05$ compared to the group with no $\mathrm{CM} ;{ }^{\dagger} p<0.05$ compared to the DCM group; ${ }^{\ddagger} p<0.05$ compared to the HCM group.

Table 3. BAV and Aorta Characteristics according to the Presence of Specific Cardiomyopathy

\begin{tabular}{|c|c|c|c|c|c|}
\hline & No CM (n=1119) & DCM $(n=10)$ & HCM (n=17) & LVNC ( $n=40)$ & $p$ value \\
\hline \multicolumn{6}{|l|}{ BAV phenotypes } \\
\hline Type 1 (RCC+LCC) & $713(64)$ & $7(70)$ & $10(56)$ & $26(65)$ & 0.934 \\
\hline Type 2 (RCC+NCC) & $192(17)$ & $1(10)$ & $2(11)$ & $8(20)$ & 0.939 \\
\hline Type 3 (LCC+NCC) & $62(6)$ & $1(10)$ & $0(0)$ & $2(5)$ & 0.653 \\
\hline Type 0 (True) & $150(13)$ & $1(10)$ & $6(30)$ & $4(10)$ & 0.725 \\
\hline \multicolumn{6}{|l|}{ BAV dysfunction } \\
\hline No or mild dysfunction & $383(34)$ & $6(60)^{\dagger}$ & $5(28)$ & $7(18)^{*+}$ & 0.048 \\
\hline Significant AS & $481(43)$ & $3(30)$ & $9(53)$ & $23(58)$ & 0.080 \\
\hline Significant AR & $354(32)$ & $2(20)$ & $6(35)$ & $16(40)$ & 0.308 \\
\hline AV area by C.E. $\left(\mathrm{cm}^{2}\right)$ & $1.60 \pm 0.35$ & $1.69 \pm 0.34$ & $1.50 \pm 0.35$ & $1.57 \pm 0.36$ & 0.249 \\
\hline AV peak gradient (mm Hg) & $13.6 \pm 8.4$ & $11.9 \pm 7.3$ & $18.5 \pm 9.5$ & $17.1 \pm 8.7$ & 0.169 \\
\hline \multicolumn{6}{|l|}{ Aorta phenotypes } \\
\hline Normal shape & $388(35)$ & $2(20)$ & $6(3)$ & $20(50)^{*}$ & 0.106 \\
\hline Predominant Valsalva sinus & $162(15)$ & $0(0)$ & $4(24)$ & $4(10)$ & 0.597 \\
\hline Predominant $\mathrm{AA}$ & $536(48)$ & $7(70)$ & $8(47)$ & $16(40)$ & 0.414 \\
\hline Presence of aortopathy & $473(42)$ & $5(50)$ & $10(59)$ & $16(40)$ & 0.808 \\
\hline AA diameter (mm) & $38.2 \pm 7.7$ & $39.3 \pm 6.1$ & $38.9 \pm 8.3$ & $37.6 \pm 9.4$ & 0.621 \\
\hline
\end{tabular}

BAV, bicuspid aortic valve; CM, cardiomyopathy; DCM, dilated cardiomyopathy; HCM, hypertrophic cardiomyopathy; LVNC, left ventricular noncompaction; RCC right coronary cusp; LCC, left coronary cusp; NCC, non-coronary cusp; AS, aortic stenosis; AR, aortic regurgitation; AV, aortic valve; C.E., continuity equation; $A A$, ascending aorta.

Values are presented as mean \pm standard deviation or $\mathrm{n}(\%)$ unless otherwise indicated.

${ }^{*} p<0.05$ compared to the group with no $\mathrm{CM} ;{ }^{\dagger} p<0.05$ compared to the DCM group. 

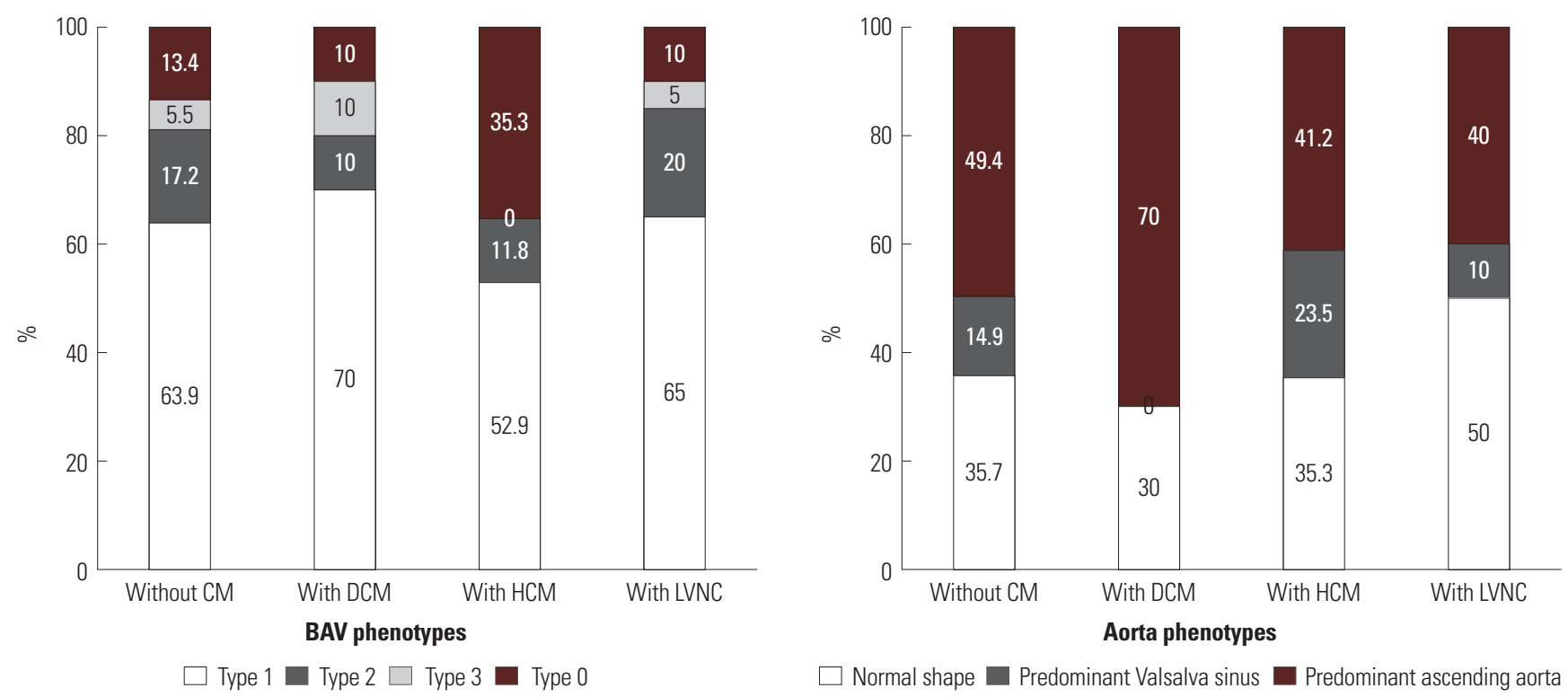

A

$\square$ Normal shape $\square$ Predominant Valsalva sinus $\square$ Predominant ascending aorta B

Fig. 2. (A) Bicuspid aortic valves (BAV) phenotypes according to the presence of specific cardiomyopathies (CMs). (B) Aorta phenotypes according to the presence of specific CMs. DCM, dilated cardiomyopathy; HCM, hypertrophic cardiomyopathy; LVNC, left ventricular non-compaction.
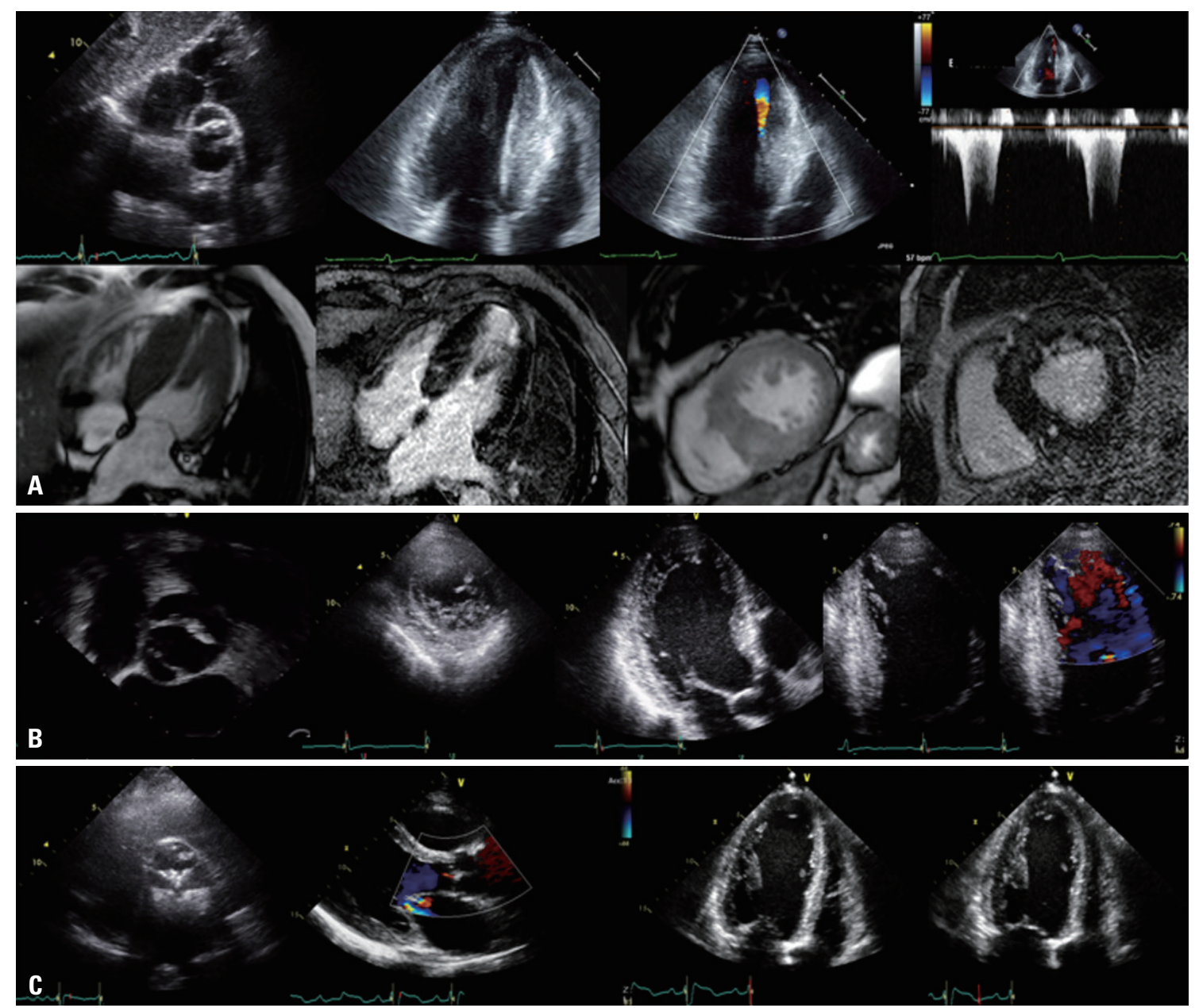

Fig. 3. Representative cases of coexisting cardiomyopathies in bicuspid aortic valves subjects. (A) Hypertrophic cardiomyopathy, (B) left ventricular noncompaction, and (C) dilated cardiomyopathy. 
ated with heart failure (Table 4). Neither the presence of aortopathy nor increased aorta diameter was independently associated with heart failure in multivariate analysis.

\section{DISCUSSION}

The principal findings of the present study are as follows. First, approximately $6 \%$ of BAV subjects had concomitant CMs. LVNC was the most prevalent; however, its prevalence in BAV subjects was only 3.4\% lower than that previously reported. Second, BAV subjects with specific CMs had distinct clinical and echocardiographic features. Third, age, hypertension, and presence of CM were significantly associated with heart failure. To the best of our knowledge, this is the first study in which the prevalence, characteristics, and clinical implications of concomitant specific CMs were identified in adults with BAV using data from a large Korean registry.

Case reports of specific CMs in BAV subjects have been sporadic, and studies on their prevalences are limited. Agarwal, et al. ${ }^{12}$ reported the incidence of LVNC in patients with BAV. In their retrospective observational study, 12 of $109 \mathrm{BAV}$ patients (11.0\%) were diagnosed with LVNC based on echocardiographic criteria. The mean age at diagnosis was $33 \pm 17$ years. The incidence was greater and mean age at diagnosis was significantly lower values thereof than in our study. Chandra, et al. ${ }^{24}$ researched the incidence of LVNC in Caucasians and AfricanAmericans, but not Asians, and revealed inter-racial differences in the frequency thereof in BAV patients. Therefore, the differences in incidences between the present study and Agarwal, et al. ${ }^{12}$ may likely be due to population characteristics, including age and race. Padang, et al. ${ }^{13}$ reported the incidence of HCM in patients with BAV. In their retrospective cohort, 23 patients were diagnosed with HCM and BAV based on echocardiographic criteria. The mean age at diagnosis was $52 \pm 16$ years. Their study showed $0.9 \%$ of patients with HCM had coexistent BAV and $0.4 \%$ of patients with BAV had coexistent HCM. The incidence of BAV in the general population is usually approxi- mately $1 \%$; therefore, the incidence of BAV was not deemed different between HCM and general populations. In our study, $1.4 \%$ of patients had concomitant BAV and HCM, and these results are similar to a previous study. ${ }^{13}$ In the present study, idiopathic DCM was identified in $0.8 \%$ of BAV subjects, similar to the results found in the general population. To date, studies on the prevalence of DCM in BAV subjects have not been published, probably because a certain degree of aortic valve dysfunction can result in chamber dilatation and myocardial dysfunction. To eliminate confounding factors, patients with significant aortic valve dysfunction were excluded when diagnosing idiopathic DCM. Therefore, the actual prevalence of coexisting DCM in BAV subjects might be underestimated in this study. In a previous study, genetic factors were suggested as a possible cause of coexistent BAV and HCM. ${ }^{25}$ However, few studies exist on genes in patients with BAV and specific CMs. In the future, genetic analysis will be important in studying the relationship between BAV and CM.

Recently, research into BAV has focused not only in the valve itself, but also in relation to the left ventricle and aorta. ${ }^{7-9}$ Since BAV patients with concomitant CMs have prominent characteristics of their own myocardial disease, there were no significantly different characteristics in BAV morphology, aortic valve dysfunction, or aorta phenotype in overall group comparison. Although type $0 \mathrm{BAV}$ phenotype and presence of aortopathy tended to be more prevalent in BAV patients with HCM, these tendencies did not show statistical significance.

Predictably, the presence of specific CMs may influence a patient's clinical course, especially in regards to heart failure in BAV subjects. Heart failure was associated with $16.9 \%$ of all BAV subjects and $32.8 \%$ of BAV subjects with specific CMs. In univariate analysis, age and well-known comorbidities were correlated with heart failure, as expected. Regarding several specific characteristics in BAV subjects, increased aortic diameter was positively associated with heart failure, while significant aortic valve dysfunction was not. These findings support a possible ventricular vascular interaction in BAV subjects, which has been suggested in previous studies. ${ }^{3,-9}$ However, the

Table 4. Factors associated with Heart Failure in BAV Subjects

\begin{tabular}{|c|c|c|c|c|c|c|}
\hline \multirow{2}{*}{ Variable } & \multicolumn{3}{|c|}{ Univariate } & \multicolumn{3}{|c|}{ Multivariate } \\
\hline & OR & $95 \% \mathrm{Cl}$ & $p$ value & OR & $95 \% \mathrm{Cl}$ & $p$ value \\
\hline Age & 1.035 & $1.002-1.047$ & $<0.001$ & 1.029 & $1.016-1.042$ & $<0.001$ \\
\hline Female & 1.164 & $0.835-1.623$ & 0.370 & 1.167 & $0.924-1.651$ & 0.384 \\
\hline Body mass index & 1.027 & 0.986-1.069 & 0.197 & 1.025 & $0.981-1.072$ & 0.273 \\
\hline Hypertension & 1.793 & $1.320-2.435$ & $<0.001$ & 1.436 & $1.034-1.195$ & 0.031 \\
\hline Diabetes mellitus & 1.704 & $1.196-2.429$ & 0.003 & 1.171 & 0.798-1.179 & 0.419 \\
\hline CKD & 2.226 & $1.420-3.489$ & $<0.001$ & 1.635 & $1.013-2.038$ & 0.044 \\
\hline Significant AS or AR & 0.958 & $0.691-1.327$ & 0.795 & 0.960 & $0.684-1.348$ & 0.815 \\
\hline Aortopathy & 1.320 & 0.973-1.790 & 0.074 & 1.194 & $0.795-1.508$ & 0.581 \\
\hline Aorta diameter & 1.020 & $1.000-1.040$ & 0.046 & - & - & - \\
\hline CM & 2.582 & $1.513-4.406$ & 0.001 & 2.795 & $1.603-4.873$ & $<0.001$ \\
\hline
\end{tabular}

BAV, bicuspid aortic valve; OR, odds ratio; $\mathrm{Cl}$, confidence interval; $\mathrm{CKD}$, chronic kidney disease; $\mathrm{AS}$, aortic stenosis; $\mathrm{AR}$, aortic regurgitation; CM, cardiomyopathy. 
strongest factor associated with heart failure was the presence of CM. Consequently, in multivariate analysis, age, hypertension, and presence of CM were independently associated with heart failure.

The present study had several limitations. First, this study included only Korean BAV subjects from a single tertiary referral center, which may result in bias. Therefore, multinational studies including various ethnic groups are needed to evaluate the prevalence of CMs in BAV subjects. However, we believe this study was the first to report on the prevalence of concomitant CMs in a large Korean registry using comprehensive reviews. Second, data were lacking regarding common genetic backgrounds in BAV patients with CMs. The results of this study may be a basis for future genetic research. Third, chronic hemodynamic effects of significant AS resulting in acquired LV hypertrophy or noncompaction cannot be fully excluded, although patients with severe AS were not included in this study. Forth, aortic diameters were measured based on echocardiographic imaging alone because only some BAV subjects underwent computed tomography or cardiac magnetic resonance imaging.

In conclusion, concomitant BAV with CMs was observed in $5.6 \%$ of our BAV population. Several clinical and echocardiographic characteristics, including comorbidities, heart failure presentation, BAV phenotypes, valve function, and presence of aortopathy were found in these patients. The presence of $\mathrm{CM}$ was independently associated with heart failure.

\section{ACKNOWLEDGEMENTS}

This study was supported in part by a faculty research grant of Yonsei University College of Medicine (6-2012-0206).

\section{AUTHOR CONTRIBUTIONS}

Conceptualization: Chi Young Shim. Data curation: Soo Youn Lee and Hyeonju Jeong. Formal analysis: Hyeonju Jeong and Chi Young Shim. Investigation: Hyeonju Jeong. Methodology: Hyeonju Jeong and Chi Young Shim. Project administration: Chi Young Shim. Resources: Hyeonju Jeong, Chi Young Shim, Darae Kim, Jah Yeon Choi, Kang-Un Choi, Soo Youn Lee, Geu-Ru Hong, and Jong-Won Ha. Software: Hyeonju Jeong and Chi Young Shim.

\section{ORCID iDs}

Hyeonju Jeong Chi Young Shim

Darae Kim

Jah Yeon Choi

Kang-Un Choi

Soo Youn Lee

Geu-Ru Hong

Jong-Won Ha

\section{REFERENCES}

1. Ward C. Clinical significance of the bicuspid aortic valve. Heart 2000;83:81-5.

2. Prakash SK, Bossé Y, Muehlschlegel JD, Michelena HI, Limongelli G, Della Corte A, et al. A roadmap to investigate the genetic basis of bicuspid aortic valve and its complications: insights from the international BAVCon (Bicuspid Aortic Valve Consortium). J Am Coll Cardiol 2014;64:832-9.

3. Shim CY, Cho IJ, Yang WI, Kang MK, Park S, Ha JW, et al. Central aortic stiffness and its association with ascending aorta dilation in subjects with a bicuspid aortic valve. J Am Soc Echocardiogr 2011;24:847-52.

4. Kim M, Shim CY, You SC, Cho IJ, Hong GR, Ha JW, et al. Characteristics of carotid artery structure and mechanical function and their relationships with aortopathy in patients with bicuspid aortic valves. Front Physiol 2017;8:622.

5. Niaz T, Poterucha JT, Johnson JN, Craviari C, Nienaber T, Palfreeman J, et al. Incidence, morphology, and progression of bicuspid aortic valve in pediatric and young adult subjects with coexisting congenital heart defects. Congenit Heart Dis 2017;12:261-9.

6. Niaz T, Poterucha JT, Olson TM, Johnson JN, Craviari C, Nienaber $\mathrm{T}$, et al. Characteristic morphologies of the bicuspid aortic valve in patients with genetic syndromes. J Am Soc Echocardiogr 2018;31:194-200.

7. Lee SY, Shim CY, Hong GR, Seo J, Cho I, Cho IJ, et al. Association of aortic phenotypes and mechanical function with left ventricular diastolic function in subjects with normally functioning bicuspid aortic valves and comparison to subjects with tricuspid aortic valves. Am J Cardiol 2015;116:1547-54.

8. Weismann CG, Lombardi KC, Grell BS, Northrup V, Sugeng L. Aortic stiffness and left ventricular diastolic function in children with well-functioning bicuspid aortic valves. Eur Heart J Cardiovasc Imaging 2016;17:225-30.

9. Lee SY, Shim CY, Hong GR, Cho IJ, Chang HJ, Ha JW, et al. Determinants and prognostic significance of symptomatic status in patients with moderately dysfunctional bicuspid aortic valves. PLoS One 2017;12:e0169285.

10. Feizi O, Farrer Brown G, Emanuel R. Familial study of hypertrophic cardiomyopathy and congenital aortic valve disease. Am J Cardiol 1978;41:956-64.

11. Brown PS Jr, Roberts CS, McIntosh CL, Roberts WC, Clark RE. Combined obstructive hypertrophic cardiomyopathy and stenotic congenitally bicuspid aortic valve. Am J Cardiol 1990;66:1273-5.

12. Agarwal A, Khandheria BK, Paterick TE, Treiber SC, Bush M, Tajik AJ. Left ventricular noncompaction in patients with bicuspid aortic valve. J Am Soc Echocardiogr 2013;26:1306-13.

13. Padang R, Gersh BJ, Ommen SR, Geske JB. Prevalence and impact of coexistent bicuspid aortic valve in hypertrophic cardiomyopathy. Heart Lung Circ 2018;27:33-40.

14. Lang RM, Badano LP, Mor-Avi V, Afilalo J, Armstrong A, Ernande $\mathrm{L}$, et al. Recommendations for cardiac chamber quantification by echocardiography in adults: an update from the American Society of Echocardiography and the European Association of Cardiovascular Imaging. Eur Heart J Cardiovasc Imaging 2015;16:233-70.

15. Lee SY, Shim CY, Kim D, Cho I, Hong GR, Ha JW, et al. Factors determining aortic valve dysfunction in Korean subjects with a bicuspid aortic valve. Am J Cardiol 2017;119:2049-55.

16. Roberts WC, Ko JM. Frequency by decades of unicuspid, bicuspid, and tricuspid aortic valves in adults having isolated aortic valve replacement for aortic stenosis, with or without associated aortic regurgitation. Circulation 2005;111:920-5. 
17. Zoghbi WA, Enriquez-Sarano M, Foster E, Grayburn PA, Kraft CD, Levine RA, et al. Recommendations for evaluation of the severity of native valvular regurgitation with two-dimensional and Doppler echocardiography. J Am Soc Echocardiogr 2003;16:777-802.

18. Baumgartner H, Hung J, Bermejo J, Chambers JB, Evangelista A, Griffin BP, et al. Echocardiographic assessment of valve stenosis: EAE/ASE recommendations for clinical practice. J Am Soc Echocardiogr 2009;22:1-23.

19. Jenni R, Oechslin E, Schneider J, Attenhofer Jost C, Kaufmann PA. Echocardiographic and pathoanatomical characteristics of isolated left ventricular non-compaction: a step towards classification as a distinct cardiomyopathy. Heart 2001;86:666-71.

20. Chin TK, Perloff JK, Williams RG, Jue K, Mohrmann R. Isolated noncompaction of left ventricular myocardium. A study of eight cases. Circulation 1990;82:507-13.

21. Stöllberger C, Finsterer J, Blazek G. Left ventricular hypertrabeculation/noncompaction and association with additional cardiac abnormalities and neuromuscular disorders. Am J Cardiol 2002; 90:899-902.

22. Petersen SE, Timperley J, Neubauer S. Left ventricular thrombi in a patient with left ventricular non-compaction in visualisation of the rationale for anticoagulation. Heart 2005;91:e4.

23. Yancy CW, Jessup M, Bozkurt B, Butler J, Casey DE Jr, Colvin MM, et al. 2017 ACC/AHA/HFSA focused update of the 2013 ACCF/ AHA Guideline for the management of heart failure: a report of the American College of Cardiology/American Heart Association Task Force on Clinical Practice Guidelines and the Heart Failure Society of America. J Am Coll Cardiol 2017;70:776-803.

24. Chandra S, Lang RM, Nicolarsen J, Gayat E, Spencer KT, Mor-Avi $\mathrm{V}$, et al. Bicuspid aortic valve: Inter-racial difference in frequency and aortic dimensions. JACC Cardiovasc Imaging 2012;5:981-9.

25. Somerville J, McDonald L. Congenital anomalies in the heart with hypertrophic cardiomyopathy. Br Heart J 1968;30:713-22. 